MIFPA-11-25 KIAS-P11038 CAS-KITPC/ITP-273

\title{
Pseudo-Supergravity Extension of the Bosonic String
}

\author{
H. Lü ${ }^{1,2}$, C.N. Pope ${ }^{3,4}$ and Zhao-Long Wang ${ }^{5}$ \\ ${ }^{1}$ China Economics and Management Academy \\ Central University of Finance and Economics, Beijing 100081, China \\ ${ }^{2}$ Institute for Advanced Study, Shenzhen University \\ Nanhai Ave 3688, Shenzhen 518060, China \\ ${ }^{3}$ George P. 8 Cynthia Woods Mitchell Institute for Fundamental Physics and Astronomy, \\ Texas A\&M University, College Station, TX 77843, USA \\ ${ }^{4}$ DAMTP, Centre for Mathematical Sciences, Cambridge University, \\ Wilberforce Road, Cambridge CB3 OWA, UK \\ ${ }^{5}$ School of Physics, Korea Institute for Advanced Study, Seoul 130-722, Korea
}

\begin{abstract}
$\underline{\text { ABSTRACT }}$
We construct a "pseudo-supersymmetric" fermionic extension of the effective action of the bosonic string in arbitrary spacetime dimension $D$. The theory is invariant under pseudo-supersymmetry transformations up to the quadratic fermion order, which is sufficient in order to be able to derive Killing spinor equations in bosonic backgrounds, and hence to define BPS type solutions determined by a system of first-order equations. The pseudo-supersymmetric theory can be extended by coupling it to a Yang-Mills pseudosupermultiplet. This also allows us to construct " $\alpha$ ' corrections" involving quadratic curvature terms. An exponential dilaton potential term, associated with the conformal anomaly for a bosonic string outside its critical dimension, can also be pseudo-supersymmetrised.
\end{abstract}




\section{Introduction}

Supersymmetry confers many nice properties on a theory, including the existence of BPS solutions that may be easier to construct than non-supersymmetric ones; positivity properties for the energy; stability arguments; and so on. A full calculation of the invariance of the action to all necessary orders in fermions (usually, this means to quartic order) is essential in order to establish the true supersymmetry of the theory. In practice, however, for many purposes one does not need to make use of the complete expressions for the supersymmetry transformation rules in order to study interesting features of the theory. For example, when looking for bosonic solutions that are supersymmetric, it suffices just to examine the Killing spinor equations that follow by requiring that the supersymmetry transformations of the fermion fields vanish in the purely bosonic background.

Killing spinor equations do not only arise in supersymmetric theories. For example, one may look for solutions of the Einstein equations in any dimension $D$ that additionally admit a covariantly-constant spinor, $D_{\mu} \eta=0$, or a solution of the equation $D_{\mu} \eta=c \Gamma_{\mu} \eta$. This raises the possibility that one might construct a "pseudo-supersymmetric" extension of such a bosonic theory, by adding suitable fermionic terms to the bosonic action 1 The defining property of such an extension would be that the action should be invariant under pseudo-supersymmetry transformations of the bosonic and fermionic fields, provided that one works only to quadratic order in the fermionic fields. Obviously one cannot expect the invariance to work beyond the quadratic order, unless the bosonic theory happens to be one that can be supersymmetrised in the ordinary way, but nevertheless the pseudosupersymmetry can be useful for purposes such as the construction of special solutions that admit a Killing spinor.

A simple concrete example of a pseudo-supersymmetric theory is Einstein gravity in $D$ dimensions, to which one adds a "pseudo-gravitino" field $\psi_{\mu}$. Thus one may consider the Lagrangian

$$
\mathcal{L}=\sqrt{-g}\left(R+\frac{1}{2} \bar{\psi}_{\mu} \Gamma^{\mu \nu \rho} D_{\nu} \psi_{\rho}\right)
$$

for which the action is invariant, modulo terms of quartic order in fermions, under the pseudo-supersymmetry transformations

$$
\delta \psi_{\mu}=D_{\mu} \epsilon, \quad \delta e_{\mu}^{a}=\frac{1}{4} \bar{\psi}_{\mu} \Gamma^{a} \epsilon
$$

\footnotetext{
${ }^{1}$ Somewhat related ideas, in the context of scalar/gravity theories, were developed in [1, 2, 3, where it was observed that there exist non-supersymmetrisable theories of gravity coupled to scalars where nonetheless, the scalar potential could be written in terms of a "superpotential." This was developed further in [4, where the concept of "fake supersymmetry" for such scalar/gravity theories was introduced.
} 
(The spinors should be either Majorana or symplectic-Majorana according to allowed possibilities in each dimension. In the symplectic-Majorana case, the (suppressed) $S p(2)$ indices are understood to be contracted with $\epsilon_{i j}$.) The pseudo-supersymmetric solutions of this theory are Ricci-flat metrics admitting covariantly-constant spinors. It should be pointed out that the crucial property for the pseudo-supersymmetric invariance of the Lagrangian (1.1) is the following identity

$$
\Gamma^{\mu \nu \rho}\left[D_{\nu}, D_{\rho}\right] \epsilon=G_{\mu \nu} \Gamma^{\nu} \epsilon
$$

where $G_{\mu \nu}$ is the Einstein tensor. The vanishing (1.3) gives rise to the projected integrability condition for Killing spinors.

It was recently proposed that the low-energy effective action for the bosonic string in $D$ dimensions,

$$
\mathcal{L}=\sqrt{-g}\left(R-\frac{1}{2}(\partial \phi)^{2}-\frac{1}{12} e^{a \phi} H_{\mu \nu \rho} H^{\mu \nu \rho}\right)
$$

where

$$
a^{2}=\frac{8}{D-2}
$$

might provide another example of a pseudo-supersymmetrisable theory [5]. The principal motivation for this conjecture was the observation in [6] that the theory described by (1.4) admits consistent Pauli sphere reductions on $S^{3}$ or $S^{D-3}$, in which the lowerdimensional theory comprises a finite number of fields including all the gauge bosons of $S O(4)$ or $S O(D-2)$ respectively. Such consistent sphere reductions are very unusual, and most of the other known examples involve reductions of supergravity theories, such as the $S^{7}$ reduction from $D=11$, for which the existence of Killing spinors in the $\mathrm{AdS}_{4} \times S^{7}$ background plays a crucial role in the consistency proof in [7. Thus, although there obviously cannot be a true supergravity explanation for the consistent sphere reductions of the bosonic string action in a general dimension $D$, it is natural to conjecture that Killing spinors of a pseudo-supersymmetric extension of the bosonic theory may play a similar role. Preliminary results in [5], preceded by the consideration of Killing spinors in theories of gravity with antisymmetric tensors in [8], and extended to include $\mathcal{O}\left(\alpha^{\prime}\right)$ corrections in [9], conjectured a system of consistent Killing spinor equations for which a set of suitable projections of the integrability conditions implied the bosonic equation of motion of the bosonic string.

Based on the fact that the low-energy effective action of the bosonic string admits consistent Killing spinor equations [5, 9, 10], we give an explicit construction of a pseudosupergravity extension of the bosonic string action (1.4) in $D$ dimensions. We show that it 
is indeed pseudo-supersymmetric at the level of quadratic fermion terms. We also show that the theory can be extended further to include a Maxwell or Yang-Mills pseudosupermultiplet. In the special case of $D=10$ dimensions, our results reduce to the standard ones for $\mathcal{N}=1$ supergravity coupled to Maxwell or Yang-Mills [11, at the level of quadratic fermion terms. Of course in the $D=10$ case the theory admits a completion when quartic fermion terms are added, but in all other dimensions the construction will break down beyond the quadratic order.

By developing an argument given in [12, 13, 14], our results for the Einstein-Yang-Mills pseudo-supergravities can be extended further to include the addition of order $\alpha^{\prime}$ corrections proportional to the square of the Riemann tensor.

The paper is organised as follows. In section 2, we construct the pseudo-supersymmetric extension of the bosonic string action (1.4), presenting the explicit form of the action and the pseudo-supersymmetry transformation rules. In section 3, we extend these results further by adding a Maxwell or Yang-Mills gauge pseudo-supermultiplet. In section 4 we re-express our results in the string frame, and in section 5 we discuss the pseudo-supersymmetrisation of curvature-squared corrections at order $\alpha^{\prime}$. In section 6 we make a further addition to the theory, by considering the conformal anomaly term that appears in th effective action for the bosonic string in dimensions $D \neq 26$ [17]. We show that this also can be pseudosupersymmetrised. The paper ends with conclusions and discussion in section 7 . In an appendix, we review some basic facts about Majorana and symplectic-Majorana spinors in general dimensions, and the symmetries of $\Gamma$-matrix products.

\section{Pseudo-supersymmetric Extension of the Bosonic String}

Motivated by the form of the Lagrangian for ten-dimensional $\mathcal{N}=1$ supergravity [11], we make the following ansatz for the Lagrangian for a pseudo-supersymmetric generalisation to arbitrary dimension $D$ :

$$
\begin{aligned}
e^{-1} \mathcal{L}_{D}= & R-\frac{1}{2}(\partial \phi)^{2}-\frac{1}{12} e^{a \phi} H_{(3)}^{2}+\frac{1}{2} \bar{\psi}_{\mu} \Gamma^{\mu \nu \rho} D_{\nu} \psi_{\rho}+\frac{1}{2} \bar{\lambda} \not D \lambda+e_{1} \bar{\psi}_{\mu} \Gamma^{\nu} \Gamma^{\mu} \lambda \partial_{\nu} \phi \\
& +e^{\frac{1}{2} a \phi}\left[e_{2} \bar{\psi}_{\mu} \Gamma^{\mu \nu \rho \sigma \lambda} \psi_{\lambda}+e_{3} \bar{\psi}^{\nu} \Gamma^{\rho} \psi^{\lambda}+e_{4} \bar{\psi}_{\mu} \Gamma^{\nu \rho \sigma} \Gamma^{\mu} \lambda+e_{5} \bar{\lambda} \Gamma^{\nu \rho \sigma} \lambda\right] H_{\nu \rho \sigma}
\end{aligned}
$$

where $H_{(3)}=d B_{(2)}$ and the constants $e_{1}, \ldots, e_{5}$ will be determined shortly. All terms except the last occur in the ten-dimensional supergravity Lagrangian. The last term tuns out to 
be necessary in all dimensions other than ten. We then make the ansatz

$$
\begin{aligned}
\delta \psi_{\mu} & =D_{\mu} \epsilon+e^{\frac{1}{2} a \phi}\left(c_{1} \Gamma_{\mu \nu \rho \sigma} F^{\nu \rho \sigma}+c_{2} H_{\mu \nu \rho} \Gamma^{\nu \rho}\right) \epsilon, \\
\delta \lambda & =c_{3}\left(\left(\Gamma^{\mu} \partial_{\mu} \phi\right) \epsilon+\frac{a}{12} e^{\frac{1}{2} a \phi} \Gamma^{\mu \nu \rho} H_{\mu \nu \rho} \epsilon\right), \\
\delta e_{\mu}^{a} & =\frac{1}{4} \bar{\psi}_{\mu} \Gamma^{a} \epsilon, \quad \text { so } \quad \delta g_{\mu \nu}=\frac{1}{2} \bar{\psi}_{(\mu} \Gamma_{\nu)} \epsilon, \\
\delta \phi & =c_{4} \bar{\epsilon} \lambda, \\
\delta B_{\mu \nu} & =\left[c_{5} \bar{\epsilon} \Gamma_{[\mu} \psi_{\nu]}+c_{6} \bar{\epsilon} \Gamma_{\mu \nu} \lambda\right] e^{-\frac{1}{2} a \phi},
\end{aligned}
$$

for the pseudo-supersymmetry transformation rules, again motivated by the form of the transformations in the ten-dimensional case. The constants $c_{1}, \ldots, c_{6}$ will also be determined shortly. Note that since pseudo-supersymmetry is only expected to hold up to the quadratic level in fermions, we do not include any higher-order fermion terms or torsion in the ansätze.

As discussed in the appendix, the fermions are all assumed to be either Majorana or symplectic-Majorana as appropriate to the spacetime dimension and the nature of the $\Gamma$ matrix representation. It is to be understood that the fermions carry suppressed $S p(2)$ doublet indices in the symplectic-Majorana case, contracted with $\epsilon_{i j}$ in fermion bilinear terms. The symmetries and spinor types can be read off from Table 1 in the appendix.

It is now a straightforward matter to determine all the unknown constants, by requiring that the action be invariant up to the level of quadratic fermion terms. Carrying this out, we find

$$
\begin{aligned}
& e_{1}=\frac{\mathrm{i}}{2 \sqrt{2 \beta}}, \quad e_{2}=-\frac{1}{48}, \quad e_{3}=-\frac{1}{8}, \quad e_{4}=-\frac{\mathrm{i} a}{24 \sqrt{2 \beta}}, \quad e_{5}=-\frac{(D-10)}{48(D-2)}, \\
& c_{1}=\frac{1}{12(D-2)}, \quad c_{2}=-\frac{(D-4)}{8(D-2)}, \quad c_{3}=\frac{\mathrm{i} \sqrt{\beta}}{2 \sqrt{2}}, \quad c_{4}=\frac{\mathrm{i}}{2 \sqrt{2 \beta}}, \quad c_{5}=-\frac{1}{2}, \\
& c_{6}=-\frac{\mathrm{i} a}{4 \sqrt{2 \beta}} .
\end{aligned}
$$

Note that $\beta$, appearing in (A.1), is listed for each dimension and representation in Table 1 in the appendix.

\section{Addition of a Yang-Mills Pseudo-Supermultiplet}

It was shown in [9] that consistent Killing spinor equations can still be defined when YangMills fields are introduced. This implies that we can add a Yang-Mills pseudo-supermultipet to the Lagrangian (2.1). We can again gain insight into the form of the additional terms needed for the inclusion of a Yang-Mills pseudo-supermultiplet by comparing with the known 
ten-dimensional results in [11]. For convenience of notation, we shall first present the results for the case of an abelian Maxwell multiplet, and then indicate the necessary modifications for the non-abelian case. Thus we are led to propose that the Lagrangian will be given by

$$
\left(\mathcal{L}_{D} \text { with } H_{\mu \nu \rho} \rightarrow \widetilde{H}_{\mu \nu \rho}\right)+\mathcal{L}_{\text {gauge }}
$$

where

$$
\widetilde{H}_{\mu \nu \rho}=H_{\mu \nu \rho}-3 \alpha_{1} A_{[\mu} \partial_{\nu} A_{\rho]}
$$

and

$$
\begin{aligned}
e^{-1} \mathcal{L}_{\text {gauge }}=\alpha_{1}\{- & \frac{1}{4} e^{\frac{a}{2} \phi} F^{\mu \nu} F_{\mu \nu}+\frac{1}{2} \bar{\chi} \not D \chi+e_{6} e^{\frac{a}{4} \phi} F^{\rho \sigma} \bar{\chi} \Gamma^{\mu} \Gamma_{\rho \sigma} \psi_{\mu} \\
& \left.+e_{7} e^{\frac{a}{4} \phi} F_{\mu \nu} \bar{\chi} \Gamma^{\mu \nu} \lambda+e_{8} e^{\frac{a}{2} \phi} \widetilde{H}_{\mu \nu \rho} \bar{\chi} \Gamma^{\mu \nu \rho} \chi\right\}
\end{aligned}
$$

The transformation rules for the gauge field $A_{\mu}$ and the gaugino $\chi$ are taken to be

$$
\delta A_{\mu}=c_{7} e^{-\frac{a}{4} \phi} \bar{\epsilon} \Gamma_{\mu} \chi, \quad \delta \chi=c_{8} e^{\frac{a}{4} \phi} F_{\mu \nu} \Gamma^{\mu \nu} \epsilon
$$

Additionally, the previous transformation rule for $B_{\mu \nu}$ in (2.2) is now augmented by

$$
\delta_{\text {extra }} B_{\mu \nu}=\alpha_{1} c_{9} e^{-\frac{a}{4} \phi} \bar{\chi} \Gamma_{[\mu} \epsilon A_{\nu]}
$$

Requiring pseudo-supersymmetry of the enlarged system, we now find that the various constants above are given by

$$
c_{7}=2 c_{8}=-c_{9}=\frac{1}{2 \sqrt{2}}, \quad e_{6}=-\frac{1}{4 \sqrt{2}}, \quad e_{7}=\frac{\mathrm{i} a}{8 \sqrt{\beta}}, \quad e_{8}=-\frac{1}{48} .
$$

The generalisation to Yang-Mills requires replacing (3.2) by

$$
\widetilde{H}_{\mu \nu \rho}=H_{\mu \nu \rho}-3 \alpha_{1} \operatorname{tr}^{\prime}\left(A_{[\mu} \partial_{\nu} A_{\rho]}-\frac{1}{3} A_{[\mu} A_{\nu} A_{\rho]}\right)
$$

taking $F_{\mu \nu}=2 \partial_{[\mu} A_{\nu]}+\left[A_{\mu}, A_{\nu}\right]$, taking traces over all terms bilinear in gauge-algebra valued fields $A_{\mu}$ and $\chi$, and Yang-Mills covariantising the usual Lorentz-covariant derivative $D_{\mu} \chi=D_{\mu}(\omega) \chi$ to

$$
\mathcal{D}_{\mu}(\omega, A) \chi=D_{\mu}(\omega) \chi+\left[A_{\mu}, \chi\right]
$$

since $\chi$ is in the adjoint representation of the Yang-Mills group. Our results for the Maxwell multiplet agree, in ten dimensions and at the quadratic fermion level, with those in [11. The Yang-Mills generalisation can be found in [13, 14]. (Partial results for the non-abelianisation of the Maxwell multiplet can be found in [15].)

To clarify the notation, note that the Yang-Mills 1-forms are defined by $A_{(1)}=A^{I} T_{I}$, where the generators $T_{I}$ are anti-hermitian, obeying the Lie algebra $\left[T_{I}, T_{J}\right]=f^{K}{ }_{I J} T_{K}$, 
and normalized in the fundamental representation as $\operatorname{tr}\left(T_{I} T_{J}\right) \equiv \beta \delta_{I J}$. Then the trace $\operatorname{tr}^{\prime}$ is defined by $\operatorname{tr}^{\prime}=\frac{1}{\beta}$ tr. Also note that for simplicity, we have have scaled the Yang-Mills fields so that its coupling appears to be unity, but with an overall coupling $\alpha_{1}$ with the Yang-Mills Lagrangian.

\section{The Lagrangian in the String Frame}

For some purposes, it is advantageous to make a conformal scaling of the metric and work in the string frame. The required transformation of fields is given by

$$
\begin{array}{rlrl}
e_{\mu}^{a} & =e^{\frac{1}{4} a \phi} \tilde{e}_{\mu}^{a}, \quad \phi=-a \Phi, & \epsilon & =e^{\frac{1}{8} a \phi} \tilde{\epsilon}, \\
\psi_{\mu} & =e^{\frac{1}{8} a \phi}\left(\widetilde{\psi}_{\mu}-\frac{\mathrm{i} a^{2}}{8 \sqrt{\beta}} \widetilde{\Gamma}_{\mu} \tilde{\lambda}\right), & \lambda=\frac{a}{2 \sqrt{2}} e^{-\frac{1}{8} a \phi} \widetilde{\lambda}, \quad \chi=e^{-\frac{1}{8} a \phi} \tilde{\chi},
\end{array}
$$

where $\widetilde{\Gamma}_{\mu}=\tilde{e}_{\mu}^{a} \Gamma_{a}$. We find, after dropping the tildes for convenience, that in the string frame the Lagrangians (2.1) and (3.3) become

$$
\begin{aligned}
e^{-1} \mathcal{L}_{D, \mathrm{str}}= & e^{-2 \Phi}\left[R+4(\partial \Phi)^{2}-\frac{1}{12} \widetilde{H}^{2}+\frac{1}{2} \bar{\psi}_{\mu} \Gamma^{\mu \nu \rho} D_{\nu} \psi_{\rho}-\frac{1}{2} \bar{\lambda} \not D \lambda-\mathrm{i} \sqrt{\beta} \bar{\lambda} \Gamma^{\mu \nu} D_{\mu} \psi_{\nu}\right. \\
& +\bar{\psi}_{\mu} \Gamma^{\mu} \psi_{\rho} \partial^{\rho} \Phi+\frac{\mathrm{i}}{\sqrt{\beta}} \bar{\psi}_{\mu} \Gamma^{\nu} \Gamma^{\mu} \lambda \partial_{\nu} \Phi \\
+ & \left.\widetilde{H}_{\nu \rho \sigma}\left\{-\frac{1}{48} \bar{\psi}_{\mu} \Gamma^{\mu \nu \rho \sigma \lambda} \psi_{\lambda}-\frac{1}{8} \bar{\psi}^{\nu} \Gamma^{\rho} \psi^{\sigma}+\frac{1}{48} \bar{\lambda} \Gamma^{\nu \rho \sigma} \lambda+\frac{\mathrm{i}}{24 \sqrt{\beta}} \bar{\psi}_{\mu} \Gamma^{\mu \nu \rho \sigma} \lambda\right\}\right],
\end{aligned}
$$

and

$$
\begin{gathered}
e^{-1} \mathcal{L}_{\text {gauge, str }}=\alpha_{1} e^{-2 \Phi} \operatorname{tr}^{\prime}\left[-\frac{1}{4} F^{\mu \nu} F_{\mu \nu}+\frac{1}{2} \bar{\chi} \not \mathcal{D}(\omega, A) \chi-\frac{1}{4 \sqrt{2}} F^{\rho \sigma} \bar{\chi} \Gamma^{\mu} \Gamma_{\rho \sigma} \psi_{\mu}\right. \\
\left.+\frac{\mathrm{i}}{4 \sqrt{2 \beta}} F_{\mu \nu} \bar{\chi} \Gamma^{\mu \nu} \lambda-\frac{1}{48} \widetilde{H}_{\mu \nu \rho} \bar{\chi} \Gamma^{\mu \nu \rho} \chi\right]
\end{gathered}
$$

The pseudo-supersymmetry transformation rules in the string frame turn out to be, after dropping the tildes,

$$
\begin{aligned}
\delta \psi_{\mu} & =D_{\mu} \epsilon-\frac{1}{8} \widetilde{H}_{\mu \nu \rho} \Gamma^{\nu \rho} \epsilon \\
\delta \lambda & =-\mathrm{i} \sqrt{\beta}\left(\Gamma^{\mu} \partial_{\mu} \Phi-\frac{1}{12} \Gamma^{\mu \nu \rho} \widetilde{H}_{\mu \nu \rho}\right) \epsilon \\
\delta \chi & =\frac{1}{4 \sqrt{2}} F_{\mu \nu} \Gamma^{\mu \nu} \epsilon \\
\delta e_{\mu}^{a} & =\frac{1}{4} \bar{\psi}_{\mu} \Gamma^{a} \epsilon \\
\delta \Phi & =-\frac{\mathrm{i}}{8 \sqrt{\beta}} \bar{\epsilon} \lambda, \\
\delta B_{\mu \nu} & =-\frac{1}{2} \bar{\epsilon} \Gamma_{[\mu} \psi_{\nu]}-\frac{\alpha_{1}}{2 \sqrt{2}} \bar{\chi} \Gamma_{[\mu} \epsilon A_{\nu]}, \\
\delta A_{\mu} & =\frac{1}{2 \sqrt{2}} \bar{\epsilon} \Gamma_{\mu} \chi .
\end{aligned}
$$


Note that $\delta \psi_{\mu}$ may be re-expressed in terms of a torsionful connection as $\delta \psi_{\mu}=D_{\mu}\left(\omega_{-}\right) \epsilon$, where

$$
\omega_{\mu \pm}^{a b} \equiv \omega_{\mu}^{a b} \pm \frac{1}{2} \widetilde{H}_{\mu}^{a b}
$$

\section{$5 \quad$ Adding a Riemann ${ }^{2}$ Term}

It was shown in [13, 14] that having obtained the ten-dimensional action for $\mathcal{N}=1$ supergravity coupled to Yang-Mills, it becomes a straightforward matter to deduce the structure of $\mathcal{N}=1$ supergravity with a curvature-squared correction parameterised by a coefficient $\alpha$. The resulting action is supersymmetric up to the order $\alpha$. (See also [12].) We can apply the analogous argument in the case of our pseudo-supersymmetric extensions of the effective action for the $D$-dimensional bosonic string. The success is ensured by the construction of consistent Killing spinor equations with curvature-squared terms [9]. The argument is best described in the string frame.

The key observation is that the supercovariant gravitino curvature $\psi^{a b}$, defined from

$$
\psi_{\mu \nu} \equiv D_{\mu}\left(\omega_{-}\right) \psi_{\nu}-D_{\nu}\left(\omega_{-}\right) \psi_{\mu}
$$

and the torsionful connection $\omega_{\mu+}^{a b}$ transform in the same way as an $S O(D-1,1)$ Yang-Mills multiplet $\left(\chi, A_{\mu}\right)$. Thus we may make the replacements

$$
\begin{aligned}
A_{\mu} & \longrightarrow \omega_{\mu+}^{a b}, \\
F_{\mu \nu} & \longrightarrow R_{\mu \nu}^{a b}\left(\omega_{+}\right), \\
\chi & \longrightarrow \frac{1}{\sqrt{2}} \psi^{a b},
\end{aligned}
$$

in the pseudo-supersymmetric Einstein-Yang-Mills action in the string frame, and thereby obtain an action for a pseudo-supersymmetric extension of the $D$-dimensional bosonic string with a curvature-squared correction. In particular, the gaugino kinetic term $\frac{1}{2} \bar{\chi} \not \supset(\omega, A) \chi$ in (4.3) will now become

$$
\frac{1}{2} \bar{\psi}^{a b} \Gamma^{\mu} \mathcal{D}_{\mu}\left(\omega, \omega_{+}\right) \psi_{a b}
$$

where

$$
\mathcal{D}_{\mu}\left(\omega, \omega_{+}\right) \psi^{a b}=D_{\mu}(\omega) \psi^{a b}+\omega_{\mu+}^{a c} \psi_{c}{ }^{b}+\omega_{\mu+}^{b c} \psi^{a}{ }_{c} .
$$

The $R^{2}$ action is given by

$$
\begin{aligned}
e^{-1} \mathcal{L}_{R^{2}, \mathrm{str}}=\frac{1}{2} \alpha_{2} e^{-2 \Phi} & {\left[\frac{1}{4} R^{\mu \nu a b}\left(\omega_{+}\right) R_{\mu \nu a b}\left(\omega_{+}\right)-\frac{1}{4} \bar{\psi}^{a b} \Gamma^{\mu} \mathcal{D}_{\mu}\left(\omega, \omega_{+}\right) \psi_{a b}\right.} \\
+ & \frac{1}{8} R^{\rho \sigma a b}\left(\omega_{+}\right) \bar{\psi}_{a b} \Gamma^{\mu} \Gamma_{\rho \sigma} \psi_{\mu}-\frac{\mathrm{i}}{8 \sqrt{\beta}} R_{\mu \nu a b}\left(\omega_{+}\right) \bar{\psi}^{a b} \Gamma^{\mu \nu} \lambda
\end{aligned}
$$




$$
\left.+\frac{1}{96} \widetilde{H}_{\mu \nu \rho} \bar{\psi}^{a b} \Gamma^{\mu \nu \rho} \psi_{a b}\right]
$$

The Bianchi identity for the 3 -form field strength is now given by

$$
d \widetilde{H}_{(3)}=-\frac{1}{2} \alpha_{1} \operatorname{tr}^{\prime}\left(F_{(2)} \wedge F_{(2)}\right)+\frac{1}{4} \alpha_{2} R_{(2)}^{a b}\left(\omega_{+}\right) \wedge R_{a b(2)}\left(\omega_{+}\right) .
$$

Note that we have adopted the standard supergravity convention for the torsional curvature

$$
R_{(2)}^{a b}\left(\omega_{ \pm}\right)=d \omega_{ \pm}^{a b}+\omega_{ \pm}^{a c} \wedge \omega_{ \pm c}^{b}=\frac{1}{2} R_{\mu \nu}^{a b}\left(\omega_{ \pm}\right) d x^{\mu} d x^{\nu}
$$

It should be pointed out that the pseudo-supersymmetric variation of $\omega_{+}$in the curvaturesquared term together with the Chern-Simons modification of $\widetilde{H}_{(3)}$ vanishes by the leading order of the equations of motion, and hence it can be ignored at the $\alpha_{2}$ order. This property was shown in [14] for $D=10$, and in [16, 9] for general dimensions. The key to prove this is the Bianchi identity of the torsional curvature, namely

$$
\nabla_{[\mu}\left(\omega, \omega_{+}\right) R_{\nu \rho]}^{a b}\left(\omega_{+}\right)=0
$$

Together with the leading order equations of motion, we find

$$
\nabla^{\mu}\left(\omega_{-}, \omega_{+}\right)\left[e^{-2 \Phi} R_{\mu \nu}^{a b}\left(\omega_{+}\right)\right]=\mathcal{O}\left(\alpha_{2}\right) .
$$

Note that the connection in the covariant derivative is $\omega_{+}$for the tangent indices in both above equations; for spacetime indices, it is $\omega$ in (5.8) and $\omega_{-}$in (5.9). These two equations are in direct parallel to the Bianchi identity and the equation of motion for the gauge field, namely

$$
\mathcal{D}_{[\rho}(\omega, A) F_{\mu \nu]}=0, \quad \mathcal{D}^{\mu}\left(\omega_{-}, A\right)\left(e^{-2 \Phi} F_{\mu \nu}\right)=\mathcal{O}\left(\alpha_{1}\right)
$$

Thus the verification of the corresponding pesudo-supersymmetric invariance up to the $\mathcal{O}\left(\alpha_{2}\right)$ order is analogous to that of the gauge fields.

The bosonic Lagrangian for the $\alpha^{\prime}$ corrections was derived in [9] with the requirement that the system admit consistent Killing spinor equations. The bosonic Lagrangian is also considered in [16] in the context of T-duality.

To conclude this section, We point out that the supersymmetry invariance of the tendimensional $\mathcal{N}=1$ supergravity Lagrangian with curvature-squared term is proven in [13, 14] only up to quadratic order in fermions, as in our case in general dimensions. 


\section{Conformal Anomaly Term}

If one performs a calculation of the beta functions for the background fields for a bosonic string in a dimension other than 26 , one obtains a conformal anomaly term for the effective action [17], which in the Einstein frame takes the form

$$
-\frac{1}{2} m^{2} e^{-\frac{1}{2} a \phi} \sqrt{-g}
$$

It was shown that the existence of consistent Killing spinor equations is unspoiled by the conformal anomaly [10]. We may now examine whether this term can also be pseudosupersymmetrised.

Let us assume for now that we are considering a dimension $D$ for which $\beta$ is, or may be chosen to be, equal to -1 (see Table 1 in the appendix). We may then consider adding conformal anomaly terms to the Lagrangian, of the form

$$
e^{-1} \mathcal{L}_{c}=-\frac{1}{2} m^{2} e^{-\frac{1}{2} a \phi}+e^{-\frac{1}{4} a \phi}\left[e_{9} \bar{\psi}_{\mu} \Gamma^{\mu \nu} \psi_{\nu}+e_{10} \bar{\lambda} \lambda+e_{11} \bar{\psi}_{\mu} \Gamma^{\mu} \lambda+\alpha_{1} e_{12} \bar{\chi} \chi\right] .
$$

We also add extra terms in the fermion transformation rules, given by

$$
\delta_{\text {extra }} \psi_{\mu}=c_{10} e^{-\frac{1}{4} a \phi} \Gamma_{\mu} \epsilon, \quad \delta_{\text {extra }} \lambda=c_{11} e^{-\frac{1}{4} a \phi} \epsilon .
$$

We now find that the action, augmented with the contribution from (6.2), is pseudosupersymmetric using the augmented transformation rules, with the coefficients in (6.2) and (6.3) determined to be

$$
\begin{aligned}
e_{9} & =\frac{\mathrm{i} m}{4 \sqrt{2}}, \quad e_{10}=\frac{\mathrm{i} m(D-4)}{4 \sqrt{2}(D-2)}, \quad e_{11}=\frac{\mathrm{i} a m}{8}, \quad e_{12}=\frac{\mathrm{i} m}{4 \sqrt{2}}, \\
c_{10} & =-\frac{\mathrm{i} m}{2 \sqrt{2}(D-2)}, \quad c_{11}=\frac{\mathrm{i} a m}{8} .
\end{aligned}
$$

After transforming to the string frame using (4.1), and then dropping the tildes, we find that the conformal anomaly Lagrangian (6.2) becomes

$$
e^{-1} \mathcal{L}_{c, \mathrm{str}}=e^{-2 \Phi}\left[-\frac{1}{2} m^{2}+\frac{\mathrm{i}}{4 \sqrt{2}} m\left(\bar{\psi}_{\mu} \Gamma^{\mu \nu} \psi_{\nu}-2 \bar{\psi}_{\mu} \Gamma^{\mu} \lambda-\bar{\lambda} \lambda+\alpha_{1} \bar{\chi} \chi\right)\right] .
$$

The transformation rules (6.3) in the string frame are now given by

$$
\delta_{\text {extra }} \psi_{\mu}=0, \quad \delta_{\text {extra }} \lambda=\frac{\mathrm{i} \sqrt{2}}{4} m \epsilon
$$

Note that in the above derivation, we excluded the Lagrangian for the curvature-squared term (5.5). However, the fact that $\delta \psi_{\mu}$ is unmodified by the conformal anomaly implies 
that the curvature-squared and conformal anomaly terms can be pseudo-supersymmetrised simultaneously. To include (5.5), we need to add the following term

$$
-\frac{\mathrm{i} \alpha_{2}}{8 \sqrt{2}} e^{-2 \Phi} \sqrt{-g} \bar{\psi}^{a b} \psi_{a b}
$$

to the Lagrangian $\mathcal{L}_{c, \text { str }}$.

In situations where $\beta=+1$, the terms in (6.2) with coefficients $e_{9}$ and $e_{10}$ will vanish identically, if the spinors are Majorana or symplectic-Majorana. In these cases, one can

still pseudo-supersymmetrise the conformal anomaly term if one doubles the number of fermions, by adding an additional doublet index,

$$
\psi_{\mu} \longrightarrow \psi_{\mu}^{\alpha}, \quad \lambda \longrightarrow \lambda^{\alpha}
$$

All the previous fermion bilinears in the Lagrangian will now have $\alpha$ and $\beta$ indices contracted with $\delta_{\alpha \beta}$. The terms in $\mathcal{L}_{c}$, on the other hand, will have the $\alpha$ and $\beta$ indices contracted with $\epsilon_{\alpha \beta}$. An $\epsilon_{\alpha \beta}$ should also be inserted in the extra terms (6.3) in transformation rules for $\psi_{\mu}$ and $\lambda$.

\section{Conclusions}

We have introduced the notion of a pseudo-supersymmetric theory, meaning a theory which is invariant under supersymmetry-like transformations provided that one works only up to the quadratic order in fermions. The symmetry could not be extended beyond the quadratic level, except in those cases where the theory happens in fact to be supersymmetric in the usual sense. A simple example of a class of pseudosupersymmetric theories is Einstein gravity in an arbitrary dimension $D$, to which one adds a "gravitino" kinetic term, as in equation (1.1).

One can do many of the things with a pseudo-supersymmetric theory that one normally does with a genuinely supersymmetric one, including defining bosonic BPS solutions as those for which there exists one or more Killing spinors for which the fermion variations vanish. For example, in the case of pseudo-supersymmetrised Einstein gravity, the BPS solutions will be Ricci-flat metrics for which the pseudo-gravitino variation given by (1.2) vanishes. In other words, the BPS solutions in this case will be Ricci-flat spaces of special holonomy, where there exists one or more covariantly-constant spinors. As well as providing a characterisation of solutions with additional structures analogous to special holonomy, imposing the pseudo-supersymmetry requirement on bosonic backgrounds can also make it 
easier to construct the solutions, since it implies that the bosonic fields will be constrained by systems of first-order differential equations.

In this paper, we have constructed a pseudo-supersymmetric fermionic extension of the low-energy effective Lagrangian (1.4) for the bosonic string. Only in ten dimensions, it would be possible to extend the pseudo-supersymmetry to become an exact supersymmetry, by adding the necessary higher-order fermion terms that would recover the standard $\mathcal{N}=1$, $D=10$ supergravity of [11. We then showed that one can couple a Maxwell or YangMills pseudo-supermultiplet to the pseudo-supergravity in arbitrary dimensions. Using an observation made in [12, 13, 14, we then showed how the Yang-Mills result can be used in order to pseudo-supersymmetrise a curvature-squared $\alpha^{\prime}$ correction to the theory, giving a theory that is pseudo-supersymmetric up to the $\alpha^{\prime}$ order. We furthermore showed that dilaton potential term that arises as the conformal anomaly if the bosonic string is embedded in dimensions other than 26 can be pseudo-supersymmetrised in an arbitrary dimension $D$.

Although it is possible to pseudo-supersymmetrise a considerably wider class of bosonic theories than those that can be genuinely supersymmetrised, it should be emphasised that it is nonetheless a highly restrictive requirement on a bosonic theory. It would be very interesting to try to gain an understanding of what the underlying properties of a bosonic theory are that allow it to be pseudo-supersymmetrised. One of the features of the lowenergy effective action of the bosonic string that led us to suspect that it might admit a pseudo-supersymmetrisation was that the theory admits remarkable Pauli sphere reductions on $S^{3}$ or $S^{D-3}$ [6]. Almost all the other known examples of consistent Pauli sphere reductions arise in supersymmetric theories. Thus there does seem to be a possible correlation between pseudo-supersymmetry and the occurrence of consistent Pauli sphere reductions. This could provide an interesting avenue for further investigation.

The successful construction of the pseudo-supergravity extension of the bosonic string in this paper suggests that pseudo-supersymmetry may be an intrinsic stringy property. It is of great interest to study at the world-sheet level whether one can construct pseudosupersymmetric string theory whose low energy effective action is pseudo-supergravity. This line of investigation may yield models that confer many desirable features of supersymmetry and yet conform with the non-supersymmetric nature of our world. 


\section{Acknowledgement}

We are grateful to Haishan Liu, Ergin Sezgin and Paul Townsend for helpful discussions. C.N.P. is grateful to the KITPC, Beijing, for hospitality during the course of this work. The research of C.N.P. is supported in part by DOE grant DE-FG03-95ER40917.

\section{A $\quad \Gamma$ Matrices and Spinors in Dimension $D$}

Here, we record some basic results about the symmetry properties of the antisymmetric products $\Gamma^{(n)} \sim \Gamma^{\mu_{1} \cdots \mu_{n}}$ of $\Gamma$ matrices, and about the occurrence of Majorana or symplecticMajorana representations, in general dimensions $D$. We refer the reader to 18 , for further details.

In the table below, we indicate the symmetry properties of $C \Gamma^{(n)}$ for each dimension $D \bmod 8$, where $C$ is the charge conjugation matrix. The symmetry ( $\mathrm{S}=$ symmetric, $\mathrm{A}=$ antisymmetric) of $C \Gamma^{(n)}$ is always the same as that of $C \Gamma^{(n+4)}$. The column headed "Spinor" indicates whether the representation is Majorana (M) or symplectic-Majorana $(\mathrm{S}-\mathrm{M})$.

Note that the $\Gamma$-matrix products $C \Gamma^{(n)}$ for odd values of $n$ always have identical symmetry properties in all Majorana representations (i.e. $C \Gamma^{(1)}, C \Gamma^{(5)}, \ldots$ are symmetric, and $C \Gamma^{(3)}, C \Gamma^{(7)}, \ldots$ are antisymmetric), and precisely the opposite symmetry properties in all symplectic-Majorana representations (i.e. $C \Gamma^{(1)}, C \Gamma^{(5)}, \ldots$ are antisymmetric; $C \Gamma^{(3)}, C \Gamma^{(7)}, \ldots$ are symmetric). For even values of $n$, on the other hand, we have to distinguish between two possible symmetry patterns. They may be characterised by a parameter $\beta$, which is either +1 or -1 . It can be defined through the equation

$$
\Gamma_{\mu}^{T}=\beta C \Gamma_{\mu} C^{-1}
$$

The values of $\beta$ for each representation are listed in Table 1.2

In our calculations and results, we assume that all spinors are either Majorana or symplectic-Majorana, according to the allowed possibilities in each spacetime dimension $D$. Symplectic-Majorana spinors each carry a doublet $S p(2)$ index $i$, and in any fermion bilinear the two indices are contacted with the antisymmetric $S p(2)$-invariant tensor $\epsilon_{i j}$. By then suppressing the $S p(2)$ indices, certain fermionic expressions take the same form in

\footnotetext{
${ }^{2}$ In fact our $\beta=-\eta$, where $\eta= \pm 1$ is defined in [18] through $\Gamma_{\mu}^{T}=-\eta C \Gamma_{\mu} C^{-1}$. We prefer to avoid the symbol $\eta$ because it is often used to denote a Killing spinor, and also because we find the opposite sign in our definition of $\beta$ more convenient.
} 
any spacetime dimension, regardless of whether the fermions are Majorana or symplecticMajorana. For example, the pseudo-gravitino kinetic term may be written as

$$
\frac{1}{2} \bar{\psi}_{\mu} \Gamma^{\mu \nu \rho} D_{\nu} \psi_{\rho}
$$

in all cases, where, in the case of symplectic-Majorana spinors, this means $\frac{1}{2} \epsilon_{i j} \bar{\psi}_{\mu}^{i} \Gamma^{\mu \nu \rho} D_{\nu} \psi_{\rho}^{j}$. There are other terms in the pseudo-supersymmetric extension of the bosonic string whose coefficients depend on the sign of $\beta$, which can be read off on a case by case basis from the table.

\begin{tabular}{|c|c|c|c|c|c|c||c|c|}
\hline$D \bmod 8$ & $C \Gamma^{(0)}$ & $C \Gamma^{(1)}$ & $C \Gamma^{(2)}$ & $C \Gamma^{(3)}$ & $C \Gamma^{(4)}$ & $C \Gamma^{(5)}$ & Spinor & $\beta$ \\
\hline \hline 0 & $\mathrm{~S}$ & $\mathrm{~S}$ & $\mathrm{~A}$ & $\mathrm{~A}$ & $\mathrm{~S}$ & $\mathrm{~S}$ & $\mathrm{M}$ & +1 \\
& $\mathrm{~S}$ & $\mathrm{~A}$ & $\mathrm{~A}$ & $\mathrm{~S}$ & $\mathrm{~S}$ & $\mathrm{~A}$ & $\mathrm{~S}-\mathrm{M}$ & -1 \\
\hline 1 & $\mathrm{~S}$ & $\mathrm{~S}$ & $\mathrm{~A}$ & $\mathrm{~A}$ & $\mathrm{~S}$ & $\mathrm{~S}$ & $\mathrm{M}$ & +1 \\
\hline 2 & $\mathrm{~S}$ & $\mathrm{~S}$ & $\mathrm{~A}$ & $\mathrm{~A}$ & $\mathrm{~S}$ & $\mathrm{~S}$ & $\mathrm{M}$ & +1 \\
& $\mathrm{~A}$ & $\mathrm{~S}$ & $\mathrm{~S}$ & $\mathrm{~A}$ & $\mathrm{~A}$ & $\mathrm{~S}$ & $\mathrm{M}$ & -1 \\
\hline 3 & $\mathrm{~A}$ & $\mathrm{~S}$ & $\mathrm{~S}$ & $\mathrm{~A}$ & $\mathrm{~A}$ & $\mathrm{~S}$ & $\mathrm{M}$ & -1 \\
\hline 4 & $\mathrm{~A}$ & $\mathrm{~S}$ & $\mathrm{~S}$ & $\mathrm{~A}$ & $\mathrm{~A}$ & $\mathrm{~S}$ & $\mathrm{M}$ & -1 \\
& $\mathrm{~A}$ & $\mathrm{~A}$ & $\mathrm{~S}$ & $\mathrm{~S}$ & $\mathrm{~A}$ & $\mathrm{~A}$ & $\mathrm{~S}-\mathrm{M}$ & +1 \\
\hline 5 & $\mathrm{~A}$ & $\mathrm{~A}$ & $\mathrm{~S}$ & $\mathrm{~S}$ & $\mathrm{~A}$ & $\mathrm{~A}$ & $\mathrm{~S}-\mathrm{M}$ & +1 \\
\hline 6 & $\mathrm{~A}$ & $\mathrm{~A}$ & $\mathrm{~S}$ & $\mathrm{~S}$ & $\mathrm{~A}$ & $\mathrm{~A}$ & $\mathrm{~S}-\mathrm{M}$ & +1 \\
& $\mathrm{~S}$ & $\mathrm{~A}$ & $\mathrm{~A}$ & $\mathrm{~S}$ & $\mathrm{~S}$ & $\mathrm{~A}$ & $\mathrm{~S}-\mathrm{M}$ & -1 \\
\hline 7 & $\mathrm{~S}$ & $\mathrm{~A}$ & $\mathrm{~A}$ & $\mathrm{~S}$ & $\mathrm{~S}$ & $\mathrm{~A}$ & $\mathrm{~S}-\mathrm{M}$ & -1 \\
\hline
\end{tabular}

Table 1: $\Gamma$-matrix symmetries and spinor reperesentations in diverse dimensions. 


\section{References}

[1] M. Cvetič, H. Lü and C.N. Pope, Domain walls and massive gauged supergravity potentials, Class. Quant. Grav. 17, 4867 (2000), hep-th/0001002.

[2] S. Cucu, H. Lü and J.F. Vazquez-Poritz, Interpolating from $A d S_{D-2} \times S^{2}$ to $A d S_{D}$, Nucl. Phys. B677, 181 (2004), hep-th/0304022.

[3] H. Lü, C.N. Pope and J.F. Vazquez-Poritz, From AdS black holes to supersymmetric flux branes, Nucl. Phys. B709, 47 (2005), hep-th/0307001.

[4] D.Z. Freedman, C. Nunez, M. Schnabl and K. Skenderis, Fake supergravity and domain wall stability, Phys. Rev. D69, 104027 (2004), hep-th/0312055.

[5] H. Lü, C.N. Pope and Z.L. Wang, Pseudo-supersymmetry, consistent sphere reduction and Killing spinors for the bosonic string, arXiv:1105.6114 [hep-th].

[6] M. Cvetič, H. Lü and C.N. Pope, Consistent Kaluza-Klein sphere reductions, Phys. Rev. D62, 064028 (2000), hep-th/0003286.

[7] B. de Wit and H. Nicolai, The consistency of the $S^{7}$ truncation in $D=11$ supergravity, Nucl. Phys. B281, 211 (1987).

[8] H. Lü and Z.L. Wang, Pseudo-Killing spinors, pseudo-supersymmetric p-branes, bubbling and less-bubbling AdS spaces, arXiv:1103.0563 [hep-th], to appear in JHEP.

[9] H. Lü and Z.L. Wang, Killing spinors for the bosonic string, arXiv:1106.1664 [hep-th].

[10] H. Liu, H. Lü and Z.L. Wang, Killing spinors for the bosonic string and the KaluzaKlein theory with scalar potentials, arXiv:1106.4566 [hep-th].

[11] E. Bergshoeff, M. de Roo, B. de Wit and P. van Nieuwenhuizen, Ten-dimensional Maxwell-Einstein supergravity, its currents, and the issue of its auxiliary fields, Nucl. Phys. B195, 97 (1982).

[12] E. Bergshoeff, A. Salam and E.Sezgin, A Supersymmetric $R^{2}$ action in six-dimensions and torsion, Phys. Lett. B 173, 73 (1986).

[13] E. Bergsoeff and M. de Roo, Supersymmetric Chern-Simons terms in ten dimensions, Phys. Lett. B218, 210 (1989). 
[14] E.A. Bergshoeff and M. de Roo, The quartic effective action of the heterotic string and supersymmetry, Nucl. Phys. B328, 439 (1989).

[15] G.F. Chapline and N.S. Manton, Unification of Yang-Mills theory and supergravity in ten-dimensions, Phys. Lett. B120, 105 (1983).

[16] D. Andriot, Heterotic string from a higher dimensional perspective, arXiv:1102.1434 [hep-th].

[17] C.G. Callan, D. Friedan, E.J. Martinec and M.J. Perry, Strings in background fields, Nucl. Phys. B262, 593 (1985).

[18] A. Van Proeyen, Tools for supersymmetry, hep-th/9910030. 